doxorubicin group, in both the early and late phases of recurrence $(P=0.017)$.

Tumor recurrence in the early phase is thought to be the result of residual tumor or the seeding of tumor cells after TURBT. Late-phase tumors, by contrast, are thought to be independent, primary cancers. Viable BCG has been shown to persist inside bladder cancer cells for $\geq 40$ days, and BCG DNA is known to be long-lasting in the bladder wall; the authors suggest that BCG prevents recurrence by remaining effective in the bladder long after TURBT.

This study has an important limitation: by 800 days the numbers of at-risk patients were too low for meaningful analysis. Further studies are needed to assess BCG's effectiveness $>800$ days after TURBT.

Katherine Sole

Original article Hinotsu S et al. (2006) Sustained prophylactic effect of intravesical bacille Calmette-Guérin for superficial bladder cancer: a smoothed hazard analysis in a randomized prospective study. Urology 67: 545-549

\section{Partial nephrectomy might be an alternative to radical nephrectomy in pediatric RCC}

Renal cell carcinoma (RCC) is rare in pediatric patients and knowledge of long-term outcomes is limited. Partial nephrectomy (also known as nephron-sparing surgery; NSS) is an effective treatment for RCC in selected adult patients, but, because of the relatively low prevalence of pediatric RCC, NSS has not been as thoroughly examined in this population. Cook et al. retrospectively analyzed survival data from pediatric patients who underwent either NSS $(n=5)$ or radical nephrectomy $(n=10)$ for RCC at a Canadian institution between 1980 and 2005, and found that both treatments resulted in similar rates of disease-free and overall survival.

All patients who underwent NSS were alive and disease-free at the end of the follow-up period (mean 61 months). Of the 10 patients who underwent radical nephrectomy, one patient had died and one had recurrent RCC at follow-up (mean 53 months); the rest were disease-free. Across all patients, $67 \%$ of tumors were ROBSON STAGE I or II. Mean tumor size in the NSS group was $2.8 \mathrm{~cm}$, compared with $7.3 \mathrm{~cm}$ in the radical nephrectomy group.

The authors conclude that NSS is a reasonable treatment for RCC in selected pediatric patients, although these findings should be confirmed in studies involving larger patient groups - which should include patients with higher-stage and/or larger tumors-and a longer follow-up period than the current study. Chrissie Giles

Original article Cook A et al. (2006) Pediatric renal cell carcinoma: single institution 25-year case series and initial experience with partial nephrectomy. J Urol 175: 1456-1460

\section{Antibiotics to treat isolated urinary tract infections}

Quinolones have replaced sulfa drugs as the antibiotic most commonly prescribed for urinary tract infections (UTIs) in American women, according to a new study. An increase in resistance to trimethoprim-sulfamethoxazole among UTI-causing bacteria in recent years has prompted some experts to recommend that sulfa antibiotics be avoided.

In the absence of a recent nationwide review, Kallen and colleagues set out to investigate the current status of antibiotic treatment for UTIs, and to identify factors that might affect the choice of antibiotic prescribed. The team analyzed data gathered between 2000 and 2002 for two national surveys designed to estimate the characteristics of outpatient visits.

The results showed that quinolones were prescribed for UTIs more often than any other class of antibiotic, and that quinolone use increased in each successive year, although this trend did not reach statistical significance. The only significant predictors of quinolone use were patient age and geographical location-older women and women in northeastern states were most likely to be prescribed quinolones. Narrow-spectrum quinolones (particularly ciprofloxacin and levofloxacin) were prescribed more often than broadspectrum quinolones, although the latter were still prescribed in a third of UTI visits in 2002.

The authors warn that the increase in use of quinolones might be unnecessary and could cause drug-resistant bacterial strains to develop; they advise clinicians to prescribe sulfa antibiotics or narrow-spectrum quinolones, rather than broad-spectrum quinolones, for UTIs whenever possible.

\section{Tamsin Osborne}

Original article Kallen AJ et al. (2006) Current antibiotic therapy for isolated urinary tract infections in women. Arch Intern Med 166: 635-639

\section{GLOSSARY}

ROBSON STAGE

A classification scheme

for renal cancer; ranges from stage I (carcinoma confined to kidney) through stage IV (tumor has spread to local adjacent organs or distant sites) 